\title{
A note on weak approximation of minors
}

by

\author{
Piotr HAJEASZ* \\ Instytut Matematyki, Uniwersytet Warszawski \\ ul. Banacha 2, 02-097 Warszawa, Polska \\ email: hajlasz@mimuw.edu.pl
}

ABSTRACT. - Let $A_{p, q}(\Omega)$, where $\Omega \subseteq R^{n}$ is a bounded open domain, be a set of all mappings $u \in W^{1, p}\left(\Omega, R^{n}\right)$ such that adj $D u \in L^{q}$. Among other results we prove that if $n-1 \leq p<n, 1<q<n /(n-1)$, then the subclass of $A_{p, q}$ mappings, which consists of mappings with bounded $(n-1)$-dimensional image, is dense in the sequential weak topology of $A_{p, q}$. We also extend this result to other $A_{p, q}$ type spaces.

1991 MSC Primary: 28A75, 73C50. Secondary: 28A05

Key words: Nonlinear elasticity, approximation of minors, Sobolev mappings, Suslin sets.

\section{INTRODUCTION AND STATEMENT}

Let $\Omega \subseteq R^{n}$ be a bounded domain. We define

$$
A_{p, q}(\Omega)=\left\{u: \Omega \rightarrow R^{n} \mid u \in W^{1, p}\left(\Omega, R^{n}\right), \operatorname{adj} D u \in L^{q}\right\},
$$

where the matrix $\operatorname{adj} D u$ satisfies the identity $(\operatorname{det} D u) \operatorname{Id}=D u \operatorname{adj} D u$.

Spaces of this type arise in a natural way as classes on which variational functionals related to nonlinear elasticity are defined (see e.g. [1], [5], [6],

* This work was partially supported by KBN grant no. 210579101 . This research was carried on while I was visiting C.M.L.A. E.N.S. de Cachan in 1993. I want to thank for support and hospitality. 
[7] [8], [10], [16], [19], [21], [22], [24], [29]). It is very important to note that if $q>p /(n-1)$, then the space $A_{p, q}$ is not linear. The space $A_{p, q}$ is endowed both with strong and weak topology. We say that $u_{k} \rightarrow u$ strongly in $A_{p, q}$, if $u_{k} \rightarrow u$ strongly in $W^{1, p}$ and adj $D u_{k} \rightarrow \operatorname{adj} D u$ strongly in $L^{q}$; $u_{k} \rightarrow u$ weakly in $A_{p, q}$ if $u_{k} \rightarrow u$ weakly in $W^{1, p}$ and $\operatorname{adj} D u_{k} \rightarrow \operatorname{adj} D u$ weakly in $L^{q}$. All kinds of weak convergence, as usual, will be denoted by "harpoon" - in place of "arrow". Our main results are Theorem 1 and its generalisation, Theorem 3 .

THEOREM 1. - If $u \in A_{p, q}(\Omega), \Omega \subseteq R^{n}, n-1 \leq p<n, 1<q<n /(n-1)$, then there exists a sequence $u^{(\nu)} \in A_{p, q}(\Omega)$, such that values of $u^{(\nu)}$ belong to a certain bounded $(n-1)$-dimensional simplicial set (which depends on $\nu$ ) and $u^{(\nu)}-u$ weakly in $A_{p, q}(\Omega)$. In the other words the subset of $A_{p, q}$, which consists of mappings with bounded $(n-1)$-dimensional range is dense in $A_{p, q}$ in the sequential weak topology.

Remark. - Note that it is not possible to substitute in the above theorem weak convergence in $A_{p, q}$ by strong convergence. Othervise we would have (after passing to a subsequence), $D u^{(\nu)} \rightarrow D u$ a.e. and hence $0 \equiv \operatorname{det} D u^{(\nu)} \rightarrow \operatorname{det} D u$ a.e.

COROLlaRY 1. - If $u$ is as above, then there exists $u^{(\nu)} \in A_{p, q}(\Omega)$, such that $u^{(\nu)}-u$ weakly in $A_{p, q}$ and $\operatorname{det} D u^{(\nu)} \equiv 0$.

COROLLARY 2. - If $u \in A_{n-1, \frac{n}{n-1}}(\Omega)$, then for each $q<n /(n-1)$ there exists a sequence $u^{(\nu)}$, such that $u^{(\nu)} \in A_{n-1, q}(\Omega), u^{(\nu)} \rightarrow u$ weakly in $A_{n-1, q}$ and $\operatorname{det} D u^{(\nu)} \equiv 0$.

The above corollaries are in contrast with the following theorem of Müller-Qi-Yan [24].

THeOREM 2 (Müller-Qi-Yan [24, Lemma 4.1]). - Let $p \geq n-1, q \geq \frac{n}{n-1}$ and $u^{(\nu)} \rightarrow u$ weakly in $A_{p, q}(\Omega)$.

1. If $q>\frac{n}{n-1}$, then $\operatorname{det} D u^{(\nu)}-\operatorname{det} D u$ in $L^{q(n-1) / n}$.

2. If $q=\frac{n}{n-1}$ and $\operatorname{det} D u^{(\nu)} \geq 0$, then $\operatorname{det} D u^{(\nu)}-\operatorname{det} D u$ in $L^{1}(K)$ for all compact sets $K \subseteq \Omega$.

The following theorem generalizes Theorem 1 .

THEOREM 3. - If $u \in W^{1, p}\left(\Omega, R^{n}\right), p<n$, then there exists a sequence $u^{(\nu)} \in W^{1, p}\left(\Omega, R^{n}\right)$ such that values of $u^{(\nu)}$ belong to a certain bounded $[p]$-dimensional simplicial set (this set depends on $\nu$ ) and $u^{(\nu)}-u$ weakly in $W^{1, p}$. Moreover if $\operatorname{adj}_{s} D u \in L^{q_{s}}$, for some $s, q_{s}$ 
such that $1<q_{s}<([p]+1) / s$, then we can additionally obtain that $\operatorname{adj}_{s} D u^{(\nu)} \rightarrow \operatorname{adj}_{s} D u$ weakly in $L^{q_{s}}$.

Remarks. - 1) Up to the sign and order, $\operatorname{adj}_{s} D u$ is a multidimensional matrix consisting of all $s \times s$ minors of $D u$ (cf. [5]). By [p] we denote the greatest integer, less then or equal to $p$.

2) By the same reason as in the case of Theorem 1, we cannot substitute weak convergence by strong convergence.

Theorems 1 and 3 are in some sense related to the results about the approximation of Sobolev mappings between manifolds $W^{1, p}(M, N)([28]$, [3] [2], [15], [4], [13], [12]). At first sight the problem seems to be different because of a different nature of Sobolev mappings $W^{1, p}(M, N)$ and mappings $A_{p, q}(\Omega)$. However the careful study shows some deep connections between these problems. In particular the crucial (for us) method of retractions (Lemma 1) is a modification of the analogous method previously used in the context of approximation of Sobolev mappings between manifolds [4], [13].

To see further connections between the theory of $W^{1, p}(M, N)$ and $A_{p, q}(\Omega)$ mappings we refer to [14].

By $Q^{n}$ we will denote a "general" $n$-dimensional cube. By $C$ we will denote a general constant. It can change its value even in the same proof. Writting for example $C(n, p)$, we will show that this constant depends on $n$ and $p$ only.

\section{PROOFS OF THEOREMS 1 AND 3}

Before we proceed to the proofs of these theorems, we shall state and prove main technical lemma (Lemma 1 below). The following mapping is defined in $R^{n} \backslash\{x\}$ :

$$
\pi_{x, \varepsilon}(z)=\left\{\begin{array}{cl}
z & \text { when }|x-z| \geq \varepsilon \\
\frac{z-x}{|z-x|} \varepsilon+x & \text { when }|x-z| \leq \varepsilon
\end{array}\right.
$$

In other words $\pi_{x, \varepsilon}$ is a mapping which is an identity on the complement of the ball $B^{n}(x, \varepsilon)$, and which is a projection along radii onto the boundary $S^{n-1}(x, \varepsilon)$ inside the ball. Evidently $\pi_{x, \varepsilon}$ is discontinuous at $z=x$. Moreover

$$
\left|D_{z}\left(\pi_{x, \varepsilon}\right)(z)\right| \leq C(n) \frac{\varepsilon}{|z-x|},
$$

Vol. $12, \mathrm{n}^{\circ}$ 4-1995. 
for $z \in B^{n}(x, \varepsilon)$. In the sequel we will use the following notation. If $\bar{x}=\left(x_{1}, \ldots, x_{k}\right)$ is a sequence of points of $R^{n}$, then we set

$$
\pi_{\bar{x}, \varepsilon}=\pi_{x_{1}, \varepsilon} \circ \cdots \circ \pi_{x_{k}, \varepsilon} .
$$

In the proof of Theorems 1 and 3 the following lemma will play a fundamental role.

Lemma 1. - Let $u \in W^{1, p}\left(\Omega, R^{n}\right), \Omega \subseteq R^{n}, p<n$. Let $A_{1}, \ldots, A_{k} \subseteq R^{n}$ be a family of measurable sets such that $(\varepsilon / 2)^{n}<\left|A_{i}\right|<\infty$ and $\operatorname{dist}\left(A_{i}, A_{j}\right) \geq 2 \varepsilon$ for all $i \neq j$. Then for almost all $\bar{x}=\left(x_{1}, \ldots, x_{k}\right) \in$ $A_{1} \times \cdots \times A_{k}, \pi_{\bar{x}, \varepsilon} \circ u \in W^{1, p}(\Omega)$. Moreover there exists $\bar{x} \in A_{1} \times \cdots \times A_{k}$ such that

$$
\int_{\Omega}\left|D\left(\pi_{\bar{x}, \varepsilon} \circ u\right)(z)\right|^{p} d z \leq C(n, p) \int_{\Omega}|D u(z)|^{p} d z,
$$

where the constant $C(n, p)$ depends on $n$ and $p$ only.

Remark. - Assumption $\operatorname{dist}\left(A_{i}, A_{j}\right) \geq 2 \varepsilon$ guarantees that the sets $\mathcal{O}_{\varepsilon}\left(A_{i}\right)=\left\{x \in R^{n} \mid \operatorname{dist}\left(x, A_{i}\right)<\varepsilon\right\}$ are pairwise disjoint.

Before we proceed to the proof of Lemma 1 we shall be concerned with some other lemmas.

We say that $f \in \operatorname{ACL}(\Omega)$ if the function $f$ is Borel measurable and absolutely continuous on almost all lines parallel to coordinate axes. Since absolutely continuous functions are almost everywhere differentiable, $f \in \operatorname{ACL}(\Omega)$ has partial derivatives a.e. and hence the gradient $\nabla f$ is defined a.e. Now we say that $f \in \operatorname{ACL}^{p}(\Omega)$ if $f \in L^{p}(\Omega) \cap \operatorname{ACL}(\Omega)$ and $|\nabla f| \in L^{p}$. The following characterization of Sobolev space is due to Nikodym ([25], [20, Section 1.1.3]).

THEOREM 4 (Nikodym). $-W^{1, p}(\Omega)=\operatorname{ACL}^{p}(\Omega)$.

Since maybe it is not evident how to understand this theorem, we shall comment it now. This theorem states that each $\mathrm{ACL}^{p}(\Omega)$ function belongs to $W^{1, p}(\Omega)$ and the gradient $\nabla f$, which is defined a.e. for $f \in \operatorname{ACL}^{p}(\Omega)$ is just the distributional gradient. On the other hand, each element $f \in W^{1, p}(\Omega)$ (which is an equivalence class of functions equal exept the set of measure zero) admit a Borel representative, which belongs to the space $\mathrm{ACL}^{p}(\Omega)$.

LeMmA 2. - Let $f: X \rightarrow Y$ be a mapping between separable metric spaces $X$ and $Y$. If $f$ is a Borel mapping, then the graph $G_{f}=\{(x, f(x)) \mid x \in X\} \subseteq X \times Y$ is a Borel set. 
Proof. - Let $\left\{A_{i}^{(n)}\right\}_{i=1}^{\infty}$ be a family of pairwise disjoint Borel subsets of $Y$ such that $\bigcup_{i=1}^{\infty} A_{i}^{(n)}=Y$ and $\operatorname{diam} A_{i}^{(n)}<1 / n$. Then the set

$$
B_{n}=\bigcup_{i=1}^{\infty}\left(f^{-1}\left(A_{i}^{(n)}\right) \times A_{i}^{(n)}\right) \subseteq X \times Y
$$

is Borel and hence $G_{f}=\bigcap_{n=1}^{\infty} B_{n}$ is also a Borel set.

We also need the following famous theorem of Lusin and Sierpiński [17], [18], [9, Thm. 2.2.13].

TheOREM 5 (Lusin-Sierpiński). - Let $P: R^{n+k} \rightarrow R^{n}$ be an orthogonal projection. If $B \subseteq R^{n+k}$ is a Borel set, then $P(B) \subseteq R^{n}$ is $H^{n}$-measurable.

Remark. - Note that this theorem is no longer valid if we assume that $B$ is $H^{n+k}$ measurable, instead of being Borel. Note also that it is not true in general that $P(B)$ is Borel even if $B$ is Borel.

We will use the above Lusin-Sierpiński's theorem in the proof of the following "Sard's type" lemma.

LeMma 3. - Assume that $f: Q^{n} \rightarrow R^{k}$ is a Borel mapping. Then the following two conditions are equivalent:

1. For almost all segments $I \subseteq Q^{n}$, parallel to one of the coordinate axes, $H^{k}(f(I))=0$.

2. For almost all $x \in R^{k}$, the set $f^{-1}(x)$ is disjoint from almost all segments parallel to one of the coordinate axes.

Remark. - For generalizations and further results see [11], [13].

Proof. - Let $P: Q^{n} \rightarrow Q^{n-1}, P\left(x_{1}, \ldots, x_{n}\right)=\left(x_{2}, \ldots, x_{n}\right)$ be the orthogonal projection in the direction parallel to the first coordinate axis. It induces the projection $\bar{P}: Q^{n} \times R^{k} \rightarrow Q^{n-1} \times R^{k}, \bar{P}(x, y)=(P(x), y)$. By Lemma 2, the graph $G_{f} \subseteq Q^{n} \times R^{k}$ is a Borel set and hence by Theorem 5, the set $\bar{P}\left(G_{f}\right) \subseteq Q^{n-1} \times R^{k}$ is $H^{n-1} \otimes H^{k}$-measurable. Now it follows directly from Fubini's theorem that the following three conditions are equivalent.

1. $\left(H^{n-1} \otimes H^{k}\right)\left(\bar{P}\left(G_{f}\right)\right)=0$.

2. For almost all segments $I$, parallel to the axis $x_{1}, H^{k}(f(I))=0$.

3. For almost all $x \in R^{k}, f^{-1}(x)$ is disjoint from almost all segments parallel to $x_{1}$. 
Of course one can repeat the above construction with $x_{1}$ replaced by $x_{2}, x_{3}, \ldots, x_{n}$. Then the lemma follows easily.

The above lemma can be applied to $\operatorname{ACL}\left(Q^{n}, R^{k}\right)$ mappings as it follows from the following elementary observation.

LEMMA 4. - If $f:[0,1] \rightarrow R^{k}, k \geq 2$ is absolutely continuous then $H^{k}(f([0,1]))=0$.

Proof. - Othervise, applying Fubini's theorem, we would find the one dimensional slice of the set $f([0,1])$ with positive one dimensional measure, and such that this slice is the image of a certain subset of $[0,1]$ with the measure zero. This contradicts the following well known fact: If $g:[0,1] \rightarrow R$ is absolutely continuous and $E \subset[0,1], H^{1}(E)=0$ then $H^{1}(g(E))=0$ (see [27, Theorem 7.18]).

Lemmas 3 and 4 lead to the fact that if $f \in \operatorname{ACL}\left(Q^{n}, R^{k}\right)$, where $k \geq 2$, then for almost all $x \in R^{k}, f^{-1}(x)$ is disjoint from almost all lines parallel to an arbitrary coordinate axis. Hence $\pi_{x, \varepsilon} \circ f \in \operatorname{ACL}\left(Q^{n}, R^{k}\right)$ for almost all $x \in R^{k}$. Now we are in position to prove Lemma 1 .

Proof of Lemma 1. - As we have already seen, for almost all $\bar{x} \in A_{1} \times \cdots \times A_{k}, \pi_{\bar{x}, \varepsilon} \circ u \in \operatorname{ACL}(\Omega)$; hence the gradient $D\left(\pi_{\bar{x}, \varepsilon} \circ u\right)$ is defined almost everywhere and now in order to prove $\pi_{\bar{x}, \varepsilon} \circ u \in W^{1, p}(\Omega)$ it suffices to prove that $\left|D\left(\pi_{\bar{x}, \varepsilon} \circ u\right)\right| \in L^{p}(\Omega)$ (see Theorem 4).

Evidently $\pi_{\vec{x}, \varepsilon} \circ u(z)=u(z)$ for $z \in \Omega \backslash \bigcup_{i=1}^{k} u^{-1}\left(B^{n}\left(x_{i}, \varepsilon\right)\right)$ and hence $D\left(\pi_{\bar{x}, \varepsilon} \circ u\right)(z)=D u(z)$ for almost all $z \in \Omega \backslash \bigcup_{i=1}^{k} u^{-1}\left(B^{n}\left(x_{i}, \varepsilon\right)\right)$. Now it is clear that the lemma will follow if we prove the inequality

$$
\begin{aligned}
& \int_{A_{k}} \cdots \int_{A_{1}} \int_{u^{-1}}\left(\bigcup_{i=1}^{k} B^{n}\left(x_{i}, \varepsilon\right)\right) \\
& \leq C(n, p)\left|A_{1}\right| \cdots\left|A_{k}\right| \int_{\Omega}|D u(z)|^{p} d z .
\end{aligned}
$$

Note that $\left.\pi_{x_{j}, \varepsilon}\right|_{B^{n}\left(x_{i}, \varepsilon\right)}=$ id for $j \neq i$ and hence for almost all $z \in u^{-1}\left(B^{n}\left(x_{i}, \varepsilon\right)\right)$

$$
\begin{aligned}
\left|D\left(\pi_{\bar{x}, \varepsilon} \circ u\right)(z)\right| & \leq\left|D\left(\pi_{x_{i}, \varepsilon}\right)(u(z))\right||D u(z)| \\
& \leq C(n) \frac{\varepsilon}{\left|u(z)-x_{i}\right|}|D u(z)|
\end{aligned}
$$


Denoting the left hand side of (2) by $I$ we have

$$
\begin{aligned}
I \leq & C(n, p) \sum_{i=1}^{k} \int_{A_{k}} \\
& \cdots \int_{A_{1}} \int_{u^{-1}\left(B^{n}\left(x_{i}, \varepsilon\right)\right)}|D u(z)|^{p}\left(\frac{\varepsilon}{\left|u(z)-x_{i}\right|}\right)^{p} d z d x_{1} \ldots d x_{k} \\
\leq & C(n, p) \sum_{i=1}^{k} \int_{u^{-1}\left(\mathcal{O}_{\varepsilon}\left(A_{i}\right)\right)}|D u(z)|^{p}\left(\left|A_{1}\right| \cdots\left|A_{k}\right|\left(\varepsilon^{p}\left|A_{i}\right|^{-p / n}\right)\right) d z \\
\leq & C(n, p)\left|A_{1}\right| \cdots\left|A_{k}\right| \int_{\Omega}|D u(z)|^{p} d z .
\end{aligned}
$$

In the last but one step we used the estimate $\int_{A}|x|^{-p} \leq C(n, p)|A|^{1-\frac{p}{n}}$. This ends the proof of (2) and hence that of the lemma.

Proof of Theorem 1. - First we will prove the theorem under the additional assumption that the image of $u$ is bounded i.e. $u(x) \in Q^{n}$ for almost all $x \in \Omega$, where $Q^{n}$ is a certain cube. Then, as we will see, the general case will follow from the density of bounded maps in the strong $A_{p, q}$ topology.

Divide the cube $Q^{n}$ in a standard way, into $\nu^{n}$ identical, small cubes. Denote these cubes by $Q_{i}^{n, \nu}, i=1,2, \ldots, \nu^{n}$. Let $\frac{1}{2} Q_{i}^{n, \nu}$ be a cube with the same centre as $Q_{i}^{n, \nu}$ and half the edgelength. Let $x \in \frac{1}{2} Q_{i}^{n, \nu}$. We define the retraction

$$
\pi_{i, x}^{\nu}: Q^{n} \backslash\{x\} \longrightarrow Q^{n} \backslash Q_{i}^{n, \nu}
$$

as follows

$$
\pi_{i, x}^{\nu}(z)=\left\{\begin{array}{cl}
z & \text { when } z \in Q^{n} \backslash Q_{i}^{n, \nu} \\
(z-x) \theta(x, z)+x & \text { when } z \in Q_{i}^{n, \nu},
\end{array}\right.
$$

where $\theta(x, z)>0$ is such that $\pi_{i, x}^{\nu}(z) \in \partial Q_{i}^{n, \nu}$. In the other words, inside $Q_{i}^{n, \nu}, \pi_{i, x}^{\nu}$ is a retraction along radii onto $\partial Q_{i}^{n, \nu}$, with "source" at $x$. Evidently $\pi_{i, x}^{\nu}$ is bilipschitz equivalent with $\pi_{x, \varepsilon}$ type mapping (with $\varepsilon=C \nu^{-1}$ ), so we can repeate the proof of Lemma 1 , replacing $A_{i}$ by $\frac{1}{2} Q_{i}^{n, \nu}$. This leads to the following corollary: There exists $x_{i} \in \frac{1}{2} Q_{i}^{n, \nu}$ for $i=1,2, \ldots, \nu^{n}$, such that

$$
u^{(\nu)}=\pi_{1, x_{1}}^{\nu} \circ \cdots \circ \pi_{\nu^{n}, x_{\nu^{n}}}^{\nu} \circ u \in W^{1, p}(\Omega)
$$

and

$$
\int_{\Omega}\left|D u^{(\nu)}(z)\right|^{p} d z \leq C(n, p) \int_{\Omega}|D u(z)|^{p} d z .
$$


Evidently the image of $u^{(\nu)}$ is $(n-1)$-dimensional. Since for $\varphi \in C^{1}$, $|\operatorname{adj} D(\varphi \circ u)(z)| \leq|D \varphi(u(z))|^{n-1}|\operatorname{adj} D u(z)|$, then it is easy to see repeating arguments from the proof of Lemma 1 that we can choose $x_{i} \in \frac{1}{2} Q_{i}^{n, \nu}$ for $i=1,2, \ldots, \nu^{n}$ in a way, such that in addition to (4)

$$
\int_{\Omega}\left|\operatorname{adj} D u^{(\nu)}(z)\right|^{q} d z \leq C(n, q) \int_{\Omega}|\operatorname{adj} D u(z)|^{q} d z
$$

Now it suffices to prove that, after extracting a subsequence, $u^{(\nu)} \rightarrow u$ in $W^{1, p}$ and $\operatorname{adj} D u^{(\nu)} \rightarrow \operatorname{adj} D u$ in $L^{q}$. We have $\left|u(z)-u^{(\nu)}(z)\right| \leq C \nu^{-1}$ a.e., so $u^{(\nu)} \rightarrow u$ a.e. and hence in $L^{p}$ (since $u$ and $u^{(\nu)}$ are bounded).

The estimations (4), (5) show that sequences $D u^{(\nu)}$ and $\operatorname{adj} D u^{(\nu)}$ are bounded in $L^{p}$ and $L^{q}$ respectively. Hence $u^{(\nu)} \rightarrow u$ in $W^{1, p}$ and moreover after taking a subsequence $\operatorname{adj} D u^{(\nu)} \rightarrow H$ in $L^{q}$ to a certain $H \in L^{q}$. By the theorem of Ball and Reshetnyak [1], [26], [5, Chapter 4, Thm. 2.6], [16], $\operatorname{adj} D u^{(\nu)} \stackrel{*}{\longrightarrow} \operatorname{adj} D u$ in measures, and hence $\operatorname{adj} D u=H$. This completes the proof in the case of bounded maps.

Since the constants in the inequalities (4) and (5) do not depend on the size of the cube $Q^{n}$, then the theorem will follow if we prove the density of bounded maps in the strong topology of $A_{p, q}$. To this end, let $P_{R}: R^{n} \rightarrow[-R, R]^{n}$ be a retraction with a Lipschitz constant 1 ; now it is easy to see that $P_{R} \circ u \stackrel{R \rightarrow \infty}{\longrightarrow} u$ in $W^{1, p}$ and $\operatorname{adj} D\left(P_{R} \circ u\right) \stackrel{R \rightarrow \infty}{\longrightarrow} \operatorname{adj} D u$ in $L^{q}$ (in the proof of the second convergence we use an elementary inequality $\left.|\operatorname{adj} D(\varphi \circ u)| \leq|\operatorname{Lip} \varphi|^{n-1}|\operatorname{adj} D u|\right)$. This completes the proof.

Proof of Theorem 3. - By the same reason as in the proof of Theorem 1, we can assume that $u$ is bounded, i.e. $u(x) \in Q^{n}$ for a.e. $x$, where $Q^{n}$ is a fixed cube.

At the beginning we prove the first part of the theorem, i.e. we will be concerned with the weak convergence of $u^{(\nu)}$, without taking care of minors $\operatorname{adj}_{s} D u^{(\nu)}$.

In the first step, as in the proof of Theorem 1, we compose the mapping $u$ with the discontinuous retraction onto $(n-1)$-dimensional set which is the union of the boundaries of all cubes $Q_{i}^{n, \nu}, i=1,2, \ldots, \nu^{n}$.

This way we obtain ( $c f$. the proof of Theorem 1) a mapping $u_{1}^{(\nu)} \in$ $W^{1, p}\left(\Omega, \bigcup_{i=1}^{\nu^{n}} \partial Q_{i}^{n, \nu}\right)$ with

$$
\int_{\Omega}\left|D u_{1}^{(\nu)}\right|^{p} d x \leq C(n, p) \int_{\Omega}|D u|^{p} d x
$$


$\left(u_{1}^{(\nu)}\right.$ is the composition of $u$ with a discontinuous retraction described above.) Since $u_{1}^{(\nu)} \stackrel{\nu \rightarrow \infty}{\longrightarrow} u$ a.e., then it follows from (6) that $u_{1}^{(\nu)} \stackrel{\nu \rightarrow \infty}{ } u$ in $W^{1, p}$. If $p \geq n-1$, then the proof is completed. Hence we can assume that $p<n-1$. The set $\partial Q_{i}^{n, \nu}$ consists of $2 n,(n-1)$-dimensional cubes. Now we divide each such $(n-1)$-dimensional cube into a family of $\nu^{n-1}$ very small cubes (with edgelength $C \nu^{-2}$ ). This leads to the decomposition $\partial Q_{i}^{n, \nu}=\bigcup_{j=1}^{2 n \nu^{(n-1)}} Q_{i, j}^{n-1, \nu}$ and hence to the decomposition of $\bigcup_{i=1}^{\nu^{n}} \partial Q_{i}^{n, \nu}$.

Now almost the same arguments as in the proof of (6) show that we can compose the mapping $u_{1}^{(\nu)}$ with the discontinuous retraction from $\bigcup_{i=1}^{\nu^{n}} \partial Q_{i}^{n, \nu}$ onto $(n-2)$-dimensional set $\bigcup_{i, j} \partial Q_{i, j}^{n-1, \nu}$. This way we obtain the mapping $u_{2}^{(\nu)} \in W^{1, p}\left(\Omega, \bigcup_{i, j} \partial Q_{i, j}^{n-1, \nu}\right)$, with

$$
\int_{\Omega}\left|D u_{2}^{(\nu)}\right|^{p} d x \leq C(n, p) \int_{\Omega}\left|D u_{1}^{(\nu)}\right|^{p} d x \leq C^{\prime}(n, p) \int_{\Omega}|D u|^{p} d x .
$$

As above $u_{2}^{(\nu)}-u$ in $W^{1, p}$. If $p \geq n-2$, then the proof is completed. If $p<n-2$, then we can of course continue this construction and compose $u_{2}^{(\nu)}$ with retraction onto $(n-3)$-dimensional set. By induction, we can continue this construction up to the moment, we compose with retraction onto $[p]$-dimensional set. This way we get $u_{n-[p]}^{(\nu)} \in W^{1, p}(\Omega)$, with values in $[p]$-dimensional set. Moreover

$$
\int_{\Omega}\left|D u_{n-[p]}^{(\nu)}\right|^{p} d x \leq C(n, p) \int_{\Omega}|D u|^{p} d x .
$$

Since $u_{n-[p]}^{(\nu)} \stackrel{\nu \rightarrow \infty}{\longrightarrow} u$ a.e., then by (7), $u_{n-[p]}^{(\nu)} \stackrel{\nu \rightarrow \infty}{\longrightarrow} u$ in $W^{1, p}$. This ends the proof of the first part of Theorem 3.

If we know additionally that $\operatorname{adj}_{s} D u \in L^{q_{s}}$ for some $1<q_{s}<$ $([p]+1) / s$, then as in the proof of Theorem 1 , we can assume that additionally to (7), the following inequality holds

$$
\int_{\Omega}\left|\operatorname{adj}_{s} D u_{n-[p]}^{(\nu)}(x)\right|^{q_{s}} d x \leq C\left(n, s, q_{s}\right) \int_{\Omega}\left|\operatorname{adj}_{s} D u(x)\right|^{q_{s}} d x .
$$

Hence up to a subsequence, $\operatorname{adj}_{s} D u_{n-[p]}^{(\nu)} \rightarrow H$ in $L^{q_{s}}$ to a certain $H \in L^{q_{s}}$. The inequality $1<q_{s}<([p]+1) / s$ implies that $p \geq s$, hence by the theorem of Ball-Reshetnyak ( $c f$. the proof of Theorem 1), $\operatorname{adj}_{s} D u_{n-[p]}^{(\nu)} \stackrel{*}{\rightarrow} \operatorname{adj}_{s} D u$ in measures and hence $H=\operatorname{adj}_{s} D u$. This ends the proof.

Vol. 12, $\mathbf{n}^{\circ}$ 4-1995. 


\section{REFERENCES}

[1] J. Ball, Convexity conditions and existence theorems in nonlinear elasticity, Arch. Rat. Mech. Anal., Vol. 63, 1977, pp. 337-403.

[2] F. BetHuel, The approximation problem for Sobolev maps between two manifolds, Acta Math., Vol. 167, 1991, pp. 153-206.

[3] F. BETHUEL and X. ZHENG, Density of smooth functions between two manifolds in Sobolev spaces, J. Funct. Anal., Vol. 80, 1988, pp. 60-75.

[4] B. Bojarski, Geometric properties of Sobolev mappings, in: Pitman Res. Notes in Math., Vol. 211, 1989, pp. 225-241.

[5] B. Dacorogna, Direct Methods in the Calculus of Variations, Springer-Verlag 1989.

[6] M. Esteban, A direct variational approach to Skyrme's model for meson fields, Comm. Math. Phys., Vol. 105, 1986, pp. 571-591.

[7] M. Esteban, A new setting for Skyrme's problem, in: Proc. Colloque problèmes variationnels (Paris, June 1988), Boston-Basel-Stuttgart 1990.

[8] M. Esteban and S. MÜLlER, Sobolev maps with the integer degree and applications to Skyrme's problem, Proc. R. Soc. Lond., Vol. 436, 1992, pp. 197-201.

[9] H. FEDERER, Geometric Measure Theory, Springer-Verlag 1969.

[10] M. Giaquinta, G. Modica and J. SouČEK, Cartesian currents and variational problems into spheres, Annali Sc. Norm. Sup. Pisa, Vol. 16, 1989, pp. 393-485.

[11] P. HaJlasz, A Sard type theorem for Borel mappings, Colloq. Math., Vol. 67, 1994, pp. 217-221.

[12] P. HaJłasz, Approximation of Sobolev mappings, Nonlinear Anal., Vol. 22, 1994, pp. 1579-1591.

[13] P. HaJlasz, Sobolev mappings, co-area formula and related topics (preprint).

[14] P. HaJŁASZ, A note on approximation of Sobolev maps, (in preparation).

[15] F. HÉleIN, Approximation of Sobolev maps between an open set and Euclidean sphere, boundary data, and singularities, Math. Ann., Vol. 285, 1989, pp. 125-140.

[16] T. IWANIEC and A. LUTOBORSKI, Integral estimates for null lagrangians, Arch. Rat. Mech. Anal., Vol. 125, 1993, pp. 25-80.

[17] N. Lusin, Sur les ensembles analytiques, Fund. Math, Vol. 10, 1927, pp. 1-95.

[18] N. Lusin and W. Sierpiński, Sur quelques propriétés des ensembles (A), Bull. de l'Acad. de Cracovie, 1918 , p. 44.

[19] J. MaLY, $L^{p}$-approximation of Jacobians, Comm. Math. Univ. Carolinae, Vol. 32, 1991, pp. 659-666.

[20] V. MAZ'YA, Sobolev Spaces, Springer-Verlag 1985.

[21] S. MülLER, Weak continuity of determinants and nonlinear elasticity, C. R. Acad. Sci. Paris, Vol. 307, Série I, 1988, pp. 501-506.

[22] S. MULLER, Higher integrability of determinants and weak convergence in $L^{1}, J$. Reine Angew. Math., Vol. 412, 1990, pp. 20-34.

[23] S. Müller, Det=det. A remark on the distributional determinant, C. R. Acad. Sci. Paris, Vol. 313, 1990, pp. 13-17.

[24] S. MÚller, T. QI and B. S. Yan, On a new class of elastic deformations not allowing for cavitation, Ann. I.H.P. Anal. Nonl., Vol. 11, 1994, pp. 217-243.

[25] O. Nixodym, Sur une classe de fonctions considérées le problème de Dirichlet, Fund. Math., Vol. 21, 1933, pp. 129-150.

[26] Y. G. RESHETNYAK, On the stability of conformal mappings in multidimensional space, Siberian Math. J., Vol. 8, 1967, pp. 65-85.

[27] W. Rudin, Real and Complex Analysis, Third edition, Mc Graw-Hill, New York 1987.

[28] R. SCHOEN and K. UhlenBeCK, Approximation theorems for Sobolev mappings, (preprint).

[29] V. ŠveraK, Regularity properties of deformations with finite energy, Arch. Rat. Mech. Anal., Vol. 100, 1988, pp. 105-127. 